\title{
Novel prognostic markers revealed by a proteomic approach separating benign from malignant insulinomas
}

Ibrahim Alkatout ${ }^{1,13}$, Juliane Friemel ${ }^{2,13}$, Barbara Sitek ${ }^{3}$, Martin Anlauf ${ }^{4}$, Patricia A Eisenach ${ }^{5}$, Kai Stühler ${ }^{6}$, Aldo Scarpa ${ }^{7}$, Aurel Perren ${ }^{8}$, Helmut E Meyer ${ }^{3,9}$, Wolfram T Knoefel ${ }^{10}$, Günter Klöppel ${ }^{11}$ and Bence Sipos ${ }^{12}$

${ }^{1}$ Clinic of Gynecology and Obstetrics, University Hospitals Schleswig-Holstein, Kiel, Germany; ${ }^{2}$ Institute of Pathology, University of Zurich, Zurich, Switzerland; ${ }^{3}$ Medizinisches Proteom-Center, Ruhr-University Bochum, Bochum,Germany; ${ }^{4}$ Section Neuroendocrine Neoplasms, Institute of Pathology, University of Düsseldorf, Düsseldorf, Germany; ${ }^{5}$ Department of Molecular Medicine, Max-Planck Institute of Biochemistry, Martinsried, Germany; ${ }^{6}$ Molecular Proteomics Laboratory, Biologisch-Medizinisches Forschungszentrum, Heinrich-Heine-Universität, Düsseldorf, Germany; ${ }^{7}$ ARC-NET Research Center and Department of Pathology and Diagnostics, University and Hospital Trust of Verona, Verona, Italy; ${ }^{8}$ Institute of Pathology, University of Bern, Bern, Switzerland; ${ }^{9}$ Institute of Pathology, University of Tübingen, Tübingen, Germany; ${ }^{10}$ Department of General, Visceral and Pediatric Surgery, University Hospital, Düsseldorf, Germany; ${ }^{11}$ Institute of Pathology, Technical University of Munich, Munich, Germany and ${ }^{12}$ Leibniz-Institut für Analytische Wissenschaften-ISAS—e.V., Dortmund, Germany

The prognosis of pancreatic neuroendocrine tumors is related to size, histology and proliferation rate. However, this stratification needs to be refined further. We conducted a proteome study on insulinomas, a well-defined pancreatic neuroendocrine tumor entity, in order to identify proteins that can be used as biomarkers for malignancy. Based on a long follow-up, insulinomas were divided into those with metastases (malignant) and those without (benign). Microdissected cells from six benign and six malignant insulinomas were subjected to a procedure combining fluorescence dye saturation labeling with high-resolution two-dimensional gel electrophoresis. Differentially expressed proteins were identified using nano liquid chromatography-electrospray ionization/multi-stage mass spectrometry and validated by immunohistochemistry on tissue microarrays containing 62 insulinomas. Sixteen differentially regulated proteins were identified among 3000 protein spots. Immunohistochemical validation revealed that aldehyde dehydrogenase 1A1 and voltage-dependent anionselective channel protein 1 showed significantly stronger expression in malignant insulinomas than in benign insulinomas, whereas tumor protein D52 (TPD52) binding protein was expressed less strongly in malignant insulinomas than in benign insulinomas. Using multivariate analysis, low TPD52 expression was identified as a strong independent prognostic factor for both recurrence-free and overall disease-related survival. Modern Pathology (2015) 28, 69-79; doi:10.1038/modpathol.2014.82; published online 20 June 2014

The prognostic assessment of pancreatic neuroendocrine tumors has improved considerably since the introduction of World Health Organization 2010 (Bosman et $a l^{1}$ ) and the European Neuroendocrine Tumour Society staging and grading system. ${ }^{2}$

Correspondence: Professor Dr B Sipos, MD, Department of Pathology and Neuropathology, University Hospital Tübingen, Liebermeisterstrasse 8, 72076 Tübingen, Germany.

E-mail: bence.sipos@med.uni-tuebingen.de

${ }^{13}$ These authors contributed equally to this work.

Received 19 January 2014; accepted 3 April 2014; published online 20 June 2014
The most relevant prognostic factors proved to be tumor size, histologic differentiation, proliferation, mitotic rate, neural invasion, angioinvasion, gross infiltration of adjacent organs, and, finally, metastasis. ${ }^{3}$ However, despite this progress in prognostic stratification, the behavior of some pancreatic neuroendocrine tumors continues to be uncertain after resection. The identification of new prognostic biomarkers is therefore required.

Proteomics enables proteins in complex protein mixtures to be quantified and identified. Here we took advantage of this approach to study insulinomas, a well-defined group of pancreatic neuroendo- 
crine tumors. Insulinomas are solitary tumors in $85 \%$ of the cases; the remaining tumors are multiple and are associated with either multiple endocrine neoplasia 1 or insulinomatosis. ${ }^{4}$ Between 85 and $90 \%$ of solitary insulinomas are benign, and patients are cured after resection. Between 10 and 15\%, however, develop metastases, sometimes years after surgery. Malignant insulinomas are usually larger than $2 \mathrm{~cm}$ in diameter. However, in a few cases they may be smaller, making it difficult to detect their malignant potential, even with the help of all available prognostic factors. ${ }^{5}$ We therefore searched for proteins whose expression in insulinomas correlated with the clinical course of the tumors. By combining tumor microdissection, sensitive difference gel electrophoresis technique, and protein mass spectrometry as well as immunohistochemical validation, we identified three proteins as potential prognostic markers in insulinomas.

\section{Materials and methods}

Supplementary Figure 1 shows an overview of the study design.

\section{Patients and Tissues}

Pancreatic neuroendocrine tumors from 62 patients suffering from persistent hyperinsulinemic hypoglycemia were studied. All patients underwent surgery between 1975 and 2006. Their pertinent clinical data was obtained from the patients' records. Freshly frozen (Table 1a) and formalinfixed paraffin-embedded tissue samples were retrieved from archives of the Departments of Pathology of the university hospitals in Zürich (Switzerland), Düsseldorf (Germany), Kiel (Germany), and Verona (Italy). The study design was approved by the local ethics committee in Tübingen. The diagnosis was based on the World Health Organization classification of 2010 (Bosman et $a l^{1}$ ) and the Euro-

Table 1a Discovery set: clinico-pathological data of 12 patients with insulinomas

\begin{tabular}{llccl}
\hline $\begin{array}{l}\text { Biological } \\
\text { behavior }\end{array}$ & Gender & Age (years) & Size $(\mathrm{cm})$ & Metastasis \\
\hline \multirow{2}{*}{ Benign } & Male & 38 & 1.2 & No \\
& Female & 76 & 1.8 & No \\
& Male & 43 & 1.8 & No \\
& Female & 33 & 2 & No \\
& Female & 44 & 1.5 & No \\
& Female & 77 & 1.1 & No \\
& & & & \\
& Male & 65 & 3 & Liver \\
& Female & 79 & 3.5 & Lymph node \\
& Male & 61 & 4 & Liver \\
& Male & 56 & 6.5 & Lymph node \\
& Male & 41 & 3 & Lymph node \\
& Male & 74 & & \\
\hline
\end{tabular}

pean Neuroendocrine Tumour Society standard procedures. ${ }^{2}$ Table $1 \mathrm{~b}$ summarizes the clinicopathological data, including follow-up until December 2007. One of the 51 patients, whose insulinomas were considered benign, had a recurrence of hypoglycemia after surgery. None of the benign cases showed metastatic disease (medium follow-up 95 months, range 35-295). Three of the 51 patients died of diseases that were not tumor related. The mean size of the insulinomas with benign behavior was $1.5 \mathrm{~cm}$ (range $0.8-3 \mathrm{~cm}$ ). According to the proliferation rate, $43 / 51$ were neuroendocrine tumors G1 $(<2 \%$ ), while $8 / 51$ were G2 (between 2 and $6 \%$ ). Eleven insulinomas were malignant; of these, tissue from the primary tumor was available in seven cases, lymph node metastasis in only one case (multiple endocrine neoplasia 1 associated), and liver metastasis in only two cases. Eight insulinomas were already metastatic at the time of removal. The

Table 1b Validation set: clinico-pathological data of 62 patients with insulinomas

\begin{tabular}{|c|c|c|}
\hline & $\begin{array}{c}\text { Benign } \\
\text { insulinomas }\end{array}$ & $\begin{array}{l}\text { Malignant } \\
\text { insulinomas }\end{array}$ \\
\hline Age (median in years) & $49(16-79)$ & $53.9(23-82)$ \\
\hline \multicolumn{3}{|l|}{ Gender } \\
\hline Male & $16(31 \%)$ & $4(36 \%)$ \\
\hline Female & $35(69 \%)$ & $7(64 \%)$ \\
\hline \multicolumn{3}{|c|}{ Localization of primary tumor } \\
\hline Head & $15(29 \%)$ & $2(18 \%)$ \\
\hline Body & $12(24 \%)$ & $1(9 \%)$ \\
\hline Tail & $24(47 \%)$ & $5(45 \%)$ \\
\hline Multicentric & 0 & $3(28 \%)$ \\
\hline \multicolumn{3}{|l|}{ Operation } \\
\hline Enucleation & $34(67 \%)$ & 0 \\
\hline Head resection & $8(16 \%)$ & $1(9 \%)$ \\
\hline Tail resection & $9(17 \%)$ & $3(28 \%)$ \\
\hline Pancreatectomy & 0 & $2(18 \%)$ \\
\hline Plus hemihepatectomy & 0 & $4(36 \%)$ \\
\hline Palliative operation & 0 & $1(9 \%)$ \\
\hline $\begin{array}{l}\text { Tumor size (median } \\
\text { in } \mathrm{mm} \text { ) }\end{array}$ & $1.5(0.8-3.5)$ & $3.1(1.0-8.0)$ \\
\hline \multicolumn{3}{|l|}{ Proliferation rate } \\
\hline $\mathrm{G} 1 \leq 2 \%$ & $43(84 \%)$ & $6(55 \%)$ \\
\hline $\mathrm{G} 2>2 \% \leq 20 \%$ & $8(16 \%)$ & $4(36 \%)$ \\
\hline $\mathrm{G} 3>20 \%$ & & $1(9 \%)$ \\
\hline \multicolumn{3}{|l|}{ Metastases } \\
\hline Yes & 0 & 11 \\
\hline No & 51 & 0 \\
\hline \multicolumn{3}{|l|}{ Follow-up } \\
\hline Recurrence & $1(9 \%)$ & $7(64 \%)$ \\
\hline No recurrence & $50(91 \%)$ & 0 \\
\hline Not known & & $4(36 \%)$ \\
\hline \multicolumn{3}{|c|}{ Survival (median, in months) } \\
\hline Alive & $47(92 \%)$ & $2(18 \%)$ \\
\hline Death due to tumor & 0 & $5(45 \%)$ \\
\hline Tumor-unrelated death & $3(6 \%)$ & $1(9 \%)$ \\
\hline Not known & $1(2 \%)$ & $3(8 \%)$ \\
\hline
\end{tabular}


other two developed metastatic disease after surgery. Five of the eight patients with malignant insulinomas died of tumor disease (medium follow-up period 27 months, range 1-108). The mean size of the malignant insulinomas was $3 \mathrm{~cm}$ (range 1.0$8.0 \mathrm{~cm})$. Six of the eight tumors were neuroendocrine tumor G1, four tumors were G2 with a proliferation rate between 3 and $11 \%$, and one primary tumor exhibited a Ki67 rate $>20 \%$ with well-differentiated morphological features.

\section{Microdissection}

Frozen tumor tissue blocks from six benign and six malignant insulinomas (Table 1a) were serially sectioned $(10 \mu \mathrm{m})$ and stained with hematoxylin. Tumor tissue was then manually microdissected under a microscope using a sterile needle, collected in $100 \mu$ l lysis buffer (TrisHCl $30 \mathrm{mM}$; thiourea $2 \mathrm{M}$; urea $7 \mathrm{M}$; CHAPS (3-[(3-cholamidopropyl)dimethylammonio]-1-propanesulfonate) 4\%, pH 8.0), and finally sonicated on ice $(6 \times 10 \mathrm{~s}$ pulses; ultrasonic cleaner, VWR Darmstadt, Germany).

\section{Preparation of the Reference Proteome}

In order to generate the reference proteome, $100 \mathrm{mg}$ of pooled benign and malignant insulinoma tissues (Table 1a) were homogenized in liquid nitrogen in $148 \mu \mathrm{l}$ lysis buffer (TrisHCl $30 \mathrm{mM}$; thiourea $2 \mathrm{M}$; urea $7 \mathrm{M}$; CHAPS 4\%, pH 8.0) using a hand homogenizer. After thawing at room temperature, sonication $(6 \times 10 \mathrm{~s}$ pulses on ice) and centrifugation (12000 $\mathrm{g}$ for $5 \mathrm{~min}$ ) were performed. The reference proteome was used for the internal standard and for identification.

\section{Protein Labeling}

The labeling conditions were optimized as described previously. ${ }^{6}$ The cell lysates of $\sim 1000$ microdissected cells were reduced in $100 \mu$ l lysis buffer by incubation with 2 nmol Tris (2-carboxyethyl) phosphine hydrochloride (TCEP (tris(2-carboxyethyl)phosphine); Sigma) at $37^{\circ} \mathrm{C}$ in the dark for $1 \mathrm{~h}$. Saturation CyDyes (GE Healthcare, Munich, Germany) were diluted with anhydrous DMF p.a. (2 nmol/ $\mu$ l; Sigma) and $4 \mathrm{nmol}$ CyDye was added to the TCEP reduced sample. The samples were vortexed, centrifuged briefly, and left at $37^{\circ} \mathrm{C}$ in the dark for $30 \mathrm{~min}$. The internal standard $(2 \mu \mathrm{g})$ was labeled with Cy3 saturation dye and the insulinoma cells of 12 patients were labeled with Cy5 dye. The labeling reaction was stopped by adding $10 \mu \mathrm{l}$ dithiothreitol (DTT; $1.08 \mathrm{~g} / \mathrm{ml}$; BioRad) and finally, before isoelectric focusing, $10 \mu \mathrm{l}$ ampholine 2-4 (GE Healthcare) was added. The internal standard was added to each labeled microdissected sample and subjected to 2-D electrophoresis.

In order to identify proteins, $200 \mu \mathrm{g}$ of reference proteome were reduced in $50 \mu \mathrm{l}$ lysis buffer by adding 26.6 nmol TCEP. After reduction, the sample was labeled with 53.2 nmol Cy3.

\section{2-D Gel Electrophoresis}

Carrier ampholyte based isoelectric focusing (IEF) was performed in a self-made IEF chamber using tube gels $(20 \mathrm{~cm} \times 1.5 \mathrm{~mm}) .{ }^{7}$ Briefly, after running a 21.25-h voltage gradient, the ejected tube gels were incubated in equilibration buffer (125 mM Tris, 40\% (w/v) glycerol, 3\% (w/v) SDS, 65 mM DTT, pH 6.8) for $10 \mathrm{~min}$. The second dimension was performed in a Desaphor VA 300 system using polyacrylamide gels (15.2\% total acrylamide, $1.3 \%$ bisacrylamide). ${ }^{7}$ The IEF tube gels were placed onto the polyacrylamide gels $(20 \mathrm{~cm} \times 30 \mathrm{~cm} \times 1.5 \mathrm{~mm})$ and fixed using $1.0 \%(\mathrm{w} / \mathrm{v})$ agarose containing $0.01 \%(\mathrm{w} / \mathrm{v})$ bromophenol blue dye (Riedel deHaen, Seelze, Germany). For protein identification, the preparative sized gel system (IEF: $20 \mathrm{~cm} \times 1.5 \mathrm{~mm}$, SDS-polyacrylamide gel electrophoresis: $20 \mathrm{~cm} \times 30 \mathrm{~cm} \times 1.5 \mathrm{~mm}$ ) was applied under identical conditions. Silver post staining was performed after gel scanning using an mass spectrometry (MS)-compatible protocol. ${ }^{8}$

\section{Liquid Chromatography-Electrospray Ionization/ Multi-Stage Mass Spectrometry}

The protein spots of interest were excised from the gel, tryptically digested and then preconcentrated and separated in a Dionex LC Packings HPLC system (Dionex LC Packings, Idstein, Germany). Electrospray ionization-MS/MS spectra were recorded using a 4000 Q Trap (Applied Biosystems, Foster City, CA, USA) high-performance hybrid triple quadrupole/linear ion trap Liquid chromatographyMS/MS mass spectrometer. The conditions for peptide separation and for mass spectrometer measurements were applied as described elsewhere. ${ }^{9}$

\section{Scanning and Image Analysis}

For the scanning, the gels were left between the glass plates and images were acquired using a Typhoon 9400 scanner (Amersham Biosciences/GE Healthcare). Excitation wavelengths and emission filters were chosen specifically for each of the CyDyesTM according to the Typhoon user guide. Before image analysis with DeCyderTM software (Amersham Biosciences/GE Healthcare), the images were cropped with ImageQuant TM software (Amersham Biosciences/GE Healthcare). The intragel spot detection and quantification were performed using the Differential In-gel Analysis mode of the DeCyder TM software. The estimated number of spots was set to 3000. An exclusion filter was applied to remove spots with a slope $>1.6$.

\section{Construction of Tissue Microarray and Immunohistochemistry}

Tissue microarrays with two tissue spots per tumor, corresponding metastases, and matched normal 
pancreas were constructed as described previously. ${ }^{10}$ Three $\mu \mathrm{m}$-thin paraffin sections from tissue microarrays were de-paraffinized, rehydrated, and immunohistochemically stained. Before applying the primary antibody, blocking with non-immune serum was performed for $20 \mathrm{~min}$. Mouse monoclonal gelsolin antibody (clone 2, $2 \mu \mathrm{g} / \mathrm{ml}$, BD Transductino Laboratories, Cat. 610413), goat polyclonal aldehyde dehydrogenase 1 A1 (ALDH1A1) antibody $(2 \mu \mathrm{g} / \mathrm{ml}$, Santa Cruz, Cat. Sc-22589, L-15), mouse monoclonal tumor protein D52 (TPD52) binding protein (clone d1C5, 1:500; Kamiya Biomedical Company), goat polyclonal voltage-dependent anion-selective channel protein 1 (VDAC1; $2 \mu \mathrm{g} / \mathrm{ml}$, Santa Cruz, sc-32063), and mouse TARDP (Novus Biologicals, 23435-A01, 1:100) were applied as primary antibodies. The detection was performed using a Mouse Vectastain Peroxidase kit (Vectastain Elite, PK-6102, Vector Laboratories, Burlingame, CA, USA). For negative controls, the primary antibodies were omitted.

\section{Evaluation of the Immunohistochemical Stains}

The intensity of reactions was scored as 1 (mild), 2 (moderate), or 3 (strong). The proportion of positive cells was estimated in percent and divided into scores ( $<10 \%$ : 1, 10-50\%:2, 51-80\%: 3, > 80\%: 4). The final score was determined as a product of the intensity of staining and the proportion of positive cells (minimum 0, maximum 12). ${ }^{11}$

\section{Statistics}

Differences in the immunohistochemical scores of benign and malignant insulinomas or metastases were analyzed by Mann-Whitney $U$ - and Kruskal-Wallis $H$-tests. Univariate survival analysis was performed using the logrank test. Multivariable logistic regression and Cox regression analyses were performed to assess the predictive value of the markers for biological behavior of tumors. $P$-values $<0.05$ were considered to be significant. Statistical tests were performed using SPSS 10.1 software and the R package 'survival'.

\section{Results}

\section{Protein Expression Profile}

The comparison of the proteome pattern of benign and malignant insulinomas revealed 28 protein spots that consisted of differentially regulated proteins. Sixteen spots were assigned to non-redundant proteins (Supplementary Figure 2). Eight of these proteins were upregulated, in both benign and malignant insulinomas. Figure 2a, c and e show examples of quantification of protein spot intensities of ALDH1, VDAC1, and TPD52, respectively. These proteins were found to be involved in metabolic processes partly associated with neoplastic transformation (see annotations in Table 2). Five proteins-ALDH1A1, VDAC1,
TPD52, Gelsolin, and TARDBP-were selected for immunohistochemical validation.

\section{Immunohistochemical Validation}

Immunostaining for ALDH1A1 revealed mainly cytoplasmic and, to a lesser extent, nuclear labeling (Figures 1a and b). In normal islets ALDH1A1 was expressed weakly to moderately. The median ALDH1A1 expression in benign insulinomas was 2.0 (s.d. 2.65, range 0-8); in malignant primary insulinomas 8 (s.d. 3.8, range 0-9); in lymph node metastases 4 (s.d. 4, range 0-8); and in liver metastases 8.5 (s.d. 2.4, range 6-12). The analysis of ALDH1A1 expression within the groups revealed significant differences (Kruskal-Wallis, $P<0.001$ ). A comparison of the scores of benign insulinomas with those of malignant insulinomas showed that ALDH1A1 was significantly more highly expressed in malignant insulinomas than in benign insulinomas (Mann-Whitney $U$-test, $P<0.001$, Table 3a and Figure 2b). No significant differences were found between primaries of malignant insulinomas and their metastases (Mann-Whitney $U$-test, $P=0.43$ ).

Immunostaining for VDAC1 showed a cytoplasmic pattern (Figures 1c and d). Normal islets were weakly or partly moderately intensely stained. Median scores for the VDAC1 stain were as follows: benign insulinomas 4 (s.d. 2.8, range 2-12); malignant primary insulinomas 6 (s.d. 2.1, range 2-12); lymph node metastases 8 (s.d. 2.2, range 4-8); and liver metastases 9 (s.d. 3.0, range 4-12). A comparison of the scores showed that VDAC1 was significantly more highly expressed in malignant insulinomas than in benign ones (Mann-Whitney $U$-test, $P=0.04$, Table 3 a and Figure 2d). Furthermore, metastases had higher VDAC1 expression levels than primary malignant insulinomas; however, this test did not reach the defined level of significance (Mann-Whitney $U$-test, $P=0.077$ ).

Immunostainings for TPD52 exhibited a cytoplasmic pattern (Figures $1 \mathrm{e}$ and $\mathrm{f}$ ) and were strong in most islets of Langerhans. Mean scores for the TPD52 staining were as follows: benign insulinomas 12 (s.d. 1.56, range 6-12); primary malignant ones 8 (s.d. 4.5, range 0-12); lymph node metastases 4 (s.d. 2.8, range 4-9); liver metastases 4 (s.d. 3.5, range 4-12). The analysis of TPD52 expression within the groups revealed significant differences (Kruskal-Wallis, $P<0.001$ ). A comparison of the scores showed that TPD52 was significantly more abundantly expressed in benign insulinomas than in malignant ones (Mann-Whitney $U$-test, $P<0.001$, Table 3a and Figure 2f). No significant difference was found between primary malignant insulinomas and metastases (Mann-Whitney $U$-test, $P=0.86$ ). Both Gelsolin and TARDBP showed a comparable expression in benign and malignant insulinomas (Table 3a). In islets, Gelsolin and TARDBP were weakly and moderately expressed, respectively. 
Table 2 Differentially expressed proteins between benign and malignant insulinomas using 2-D gel electrophoresis

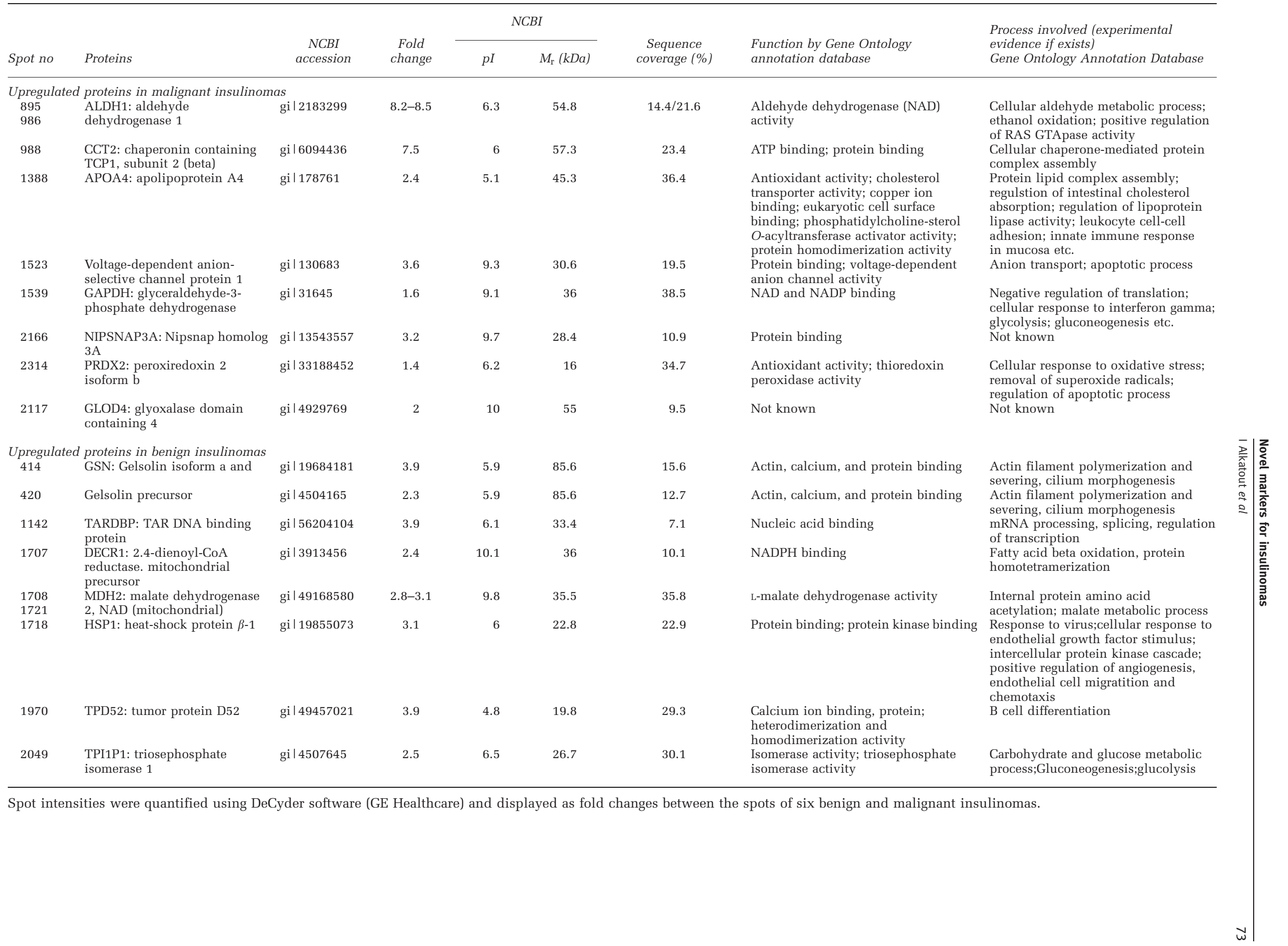


A multivariate linear regression (Table 3a) was fitted, including staining intensity scores of all potential markers as covariates, controlling for tumor size, age, and grade (grade 1 vs 2/3). TPD52 and ALDH1A1 were found to be independent predictors of malignancy with the following odds ratios: TPD52, 0.92 (95\% CI: 0.905-0.947, $P<0.0001)$ and ALDH1A1, 1.032 (95\% CI: 1.01-1.055, $P=0.0078$ ).

\section{Univariate and Multivariate Analyses Associating Biomarker Expression with Recurrence-Free and Overall Patient Outcome}

Of the 62 patient samples analyzed for staining intensity, either 1 or 2 cases were excluded due to either incomplete recurrence-free or incomplete overall survival follow-up data. Analyses of survival showed that expression of TPD52, ALDH1A1, Gelsolin, and TARDBP was significantly associated with both recurrence-free and overall survival in univariate logrank tests (Table 3B). Logistic regression analysis assessing age (as a continuous variable), proliferation (dichotomized into 0 vs 1 and 2), grade (dichotomized into 1 vs 2 and 3 ), and staining intensity scores confirmed only TPD52 as an independent predictor for recurrence-free $(\mathrm{HR}=0.66$; 95\% CI: $0.52-0.84 ; P=0.0007)$ and overall survival $(\mathrm{HR}=0.65 ; 95 \%$ CI: $0.50-0.84 ; P=0.00112)$ in the cohort analyzed. Patients with low TPD52 expression corresponded to those with a poorer outcome in recurrence-free survival $(P=3.86 \mathrm{e}-06)$ and overall survival $(P=7.71 \mathrm{e}-07$; Figure 3$)$ using Cox regression analysis.

\section{Discussion}

Studies on metastatic risk in pancreatic neuroendocrine tumors focused initially on pathological criteria, but subsequently also considered immunohistochemical markers and genetic abnormalities as prognostic factors. The most predictive factors proved to be tumor size, proliferative activity, angioinvasion, peritumoral or stromal infiltration, and tumor necrosis. ${ }^{12}$ Among the immunohistochemical markers, only cytokeratin 19 reached

Table 3a Results of immunohistochemical stains analyzed by univariate non-parametric Mann-Whitney test and by multivariate logistic regression for associations with biological behavior

Univariate Mann-Whitney

\begin{tabular}{l} 
Variables \\
\hline Age (years) \\
Size \\
Grade (1 vs 2/3) \\
TPD52 \\
VDAC1 \\
ALDH1 \\
Gelsolin \\
TARDBP
\end{tabular}

Bold entries mark significant $P$-values.

${ }^{\text {a}}$ Fisher's exact test.

bone patient is missing.

${ }^{\mathrm{C}}$ Two patients are missing.

Table 3b Results of immunohistochemistry assessed for associations with recurrence-free and overall disease-related survival using univariate and multivariate logistic regression

\begin{tabular}{|c|c|c|c|c|c|c|c|}
\hline \multirow{3}{*}{$\begin{array}{l}\text { Variable } \\
\text { All }\end{array}$} & \multirow{4}{*}{$\begin{array}{c}\text { Cases (n) } \\
60^{\mathrm{a}} \\
20 / 40\end{array}$} & \multicolumn{3}{|c|}{ Recurrence-free survival } & \multicolumn{3}{|c|}{ Overall survival } \\
\hline & & \multirow{3}{*}{$\begin{array}{c}\text { Univariate } L R \\
\text { P-value } \\
0.80\end{array}$} & \multicolumn{2}{|c|}{ Multivariate analysis } & \multirow{3}{*}{$\begin{array}{c}\text { Univariate } L R \\
\text { P-value } \\
0.84\end{array}$} & \multicolumn{2}{|c|}{ Multivariate analysis } \\
\hline & & & $P$-value & HR (5-95\%) & & $\mathrm{P}$-value & HR (5-95\%) \\
\hline Sex (male vs female) & & & & & & & \\
\hline Age (mean $\pm 95 \%$ CI) & $49.3(44.9-53.7)$ & 0.0247 & 0.29 & $1.02(0.98-1.07)$ & 0.019 & 0.18 & $1.03(0.98-1.08) 2$ \\
\hline Size $($ mean $\pm 95 \%$ CI $)$ & $1.8(1.4-2.2)$ & 0.16 & & & 0.000332 & 0.77 & $1.06(0.7-1.6)$ \\
\hline Grade (1 vs 2/3) & $47 / 13$ & 0.0138 & 0.028 & $6.3(1.22-33.02)$ & 0.019 & 0.11 & $5.31(0.7-40.4)$ \\
\hline TPD52 (mean $\pm 95 \%$ CI) & $10.3(9.5-11.1)$ & 0.0000 & 0.0007 & $0.66(0.52-0.84)$ & $1.57 \mathrm{E}-10$ & 0.001 & $0.65(0.50-0.84)$ \\
\hline VDAC1 (mean $\pm 95 \%$ CI) & $6.2(5.5-6.9)$ & 0.74 & & & 0.74 & & \\
\hline ALDH1 (mean $\pm 95 \%$ CI) & $3.5(2.6-4.3)$ & 0.0000 & 0.88 & $1.01(0.78-1.31)$ & 8.71E-05 & 0.65 & $0.93(0.70-1.24)$ \\
\hline Gelsolin (mean $\pm 95 \%$ CI) & $6.9(5.8-8.0)$ & 0.0079 & 0.78 & $0.97(0.81-1.16)$ & 0.00177 & 0.56 & $0.94(0.78-1.14)$ \\
\hline TARDBP (mean $\pm 95 \%$ CI) & $5.5(5.0-6.0)$ & 0.031 & 0.99 & $0.99(0.67-1.48)$ & 5.63E-06 & 0.94 & $1.01(0.6-1.58)$ \\
\hline
\end{tabular}

Bold entries mark significant $P$-values.

${ }^{a}$ Two patients excluded due to lack of recurrence-free survival. 


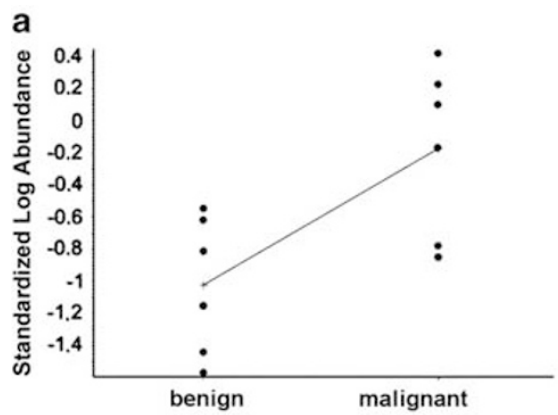

b
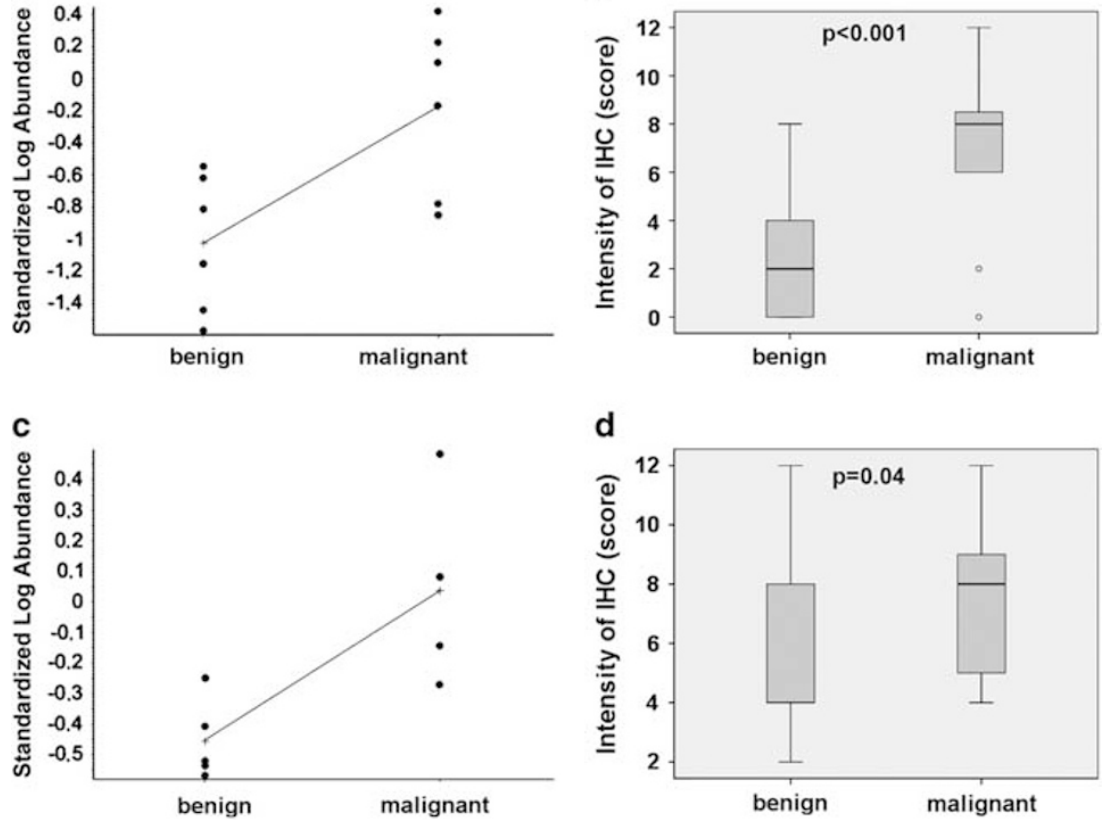

d
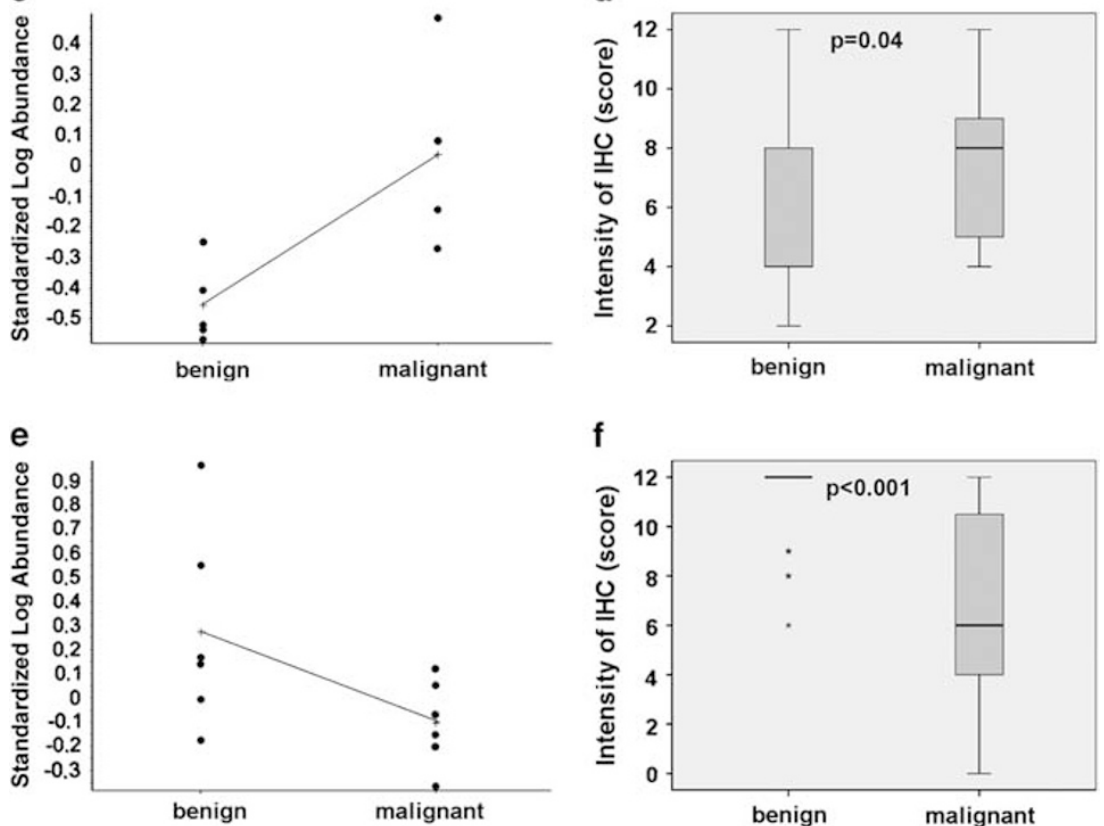

f

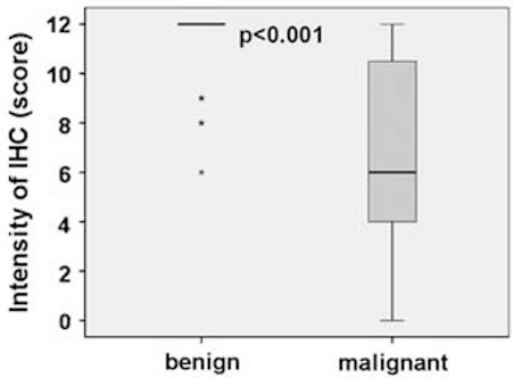

Figure 1 Representative immunohistochemical stains of aldehyde dehydrogenase 1A1 (ALDH1A1) in a benign (a) and malignant (b) insulinoma $(\times 200)$; voltage-dependent anion-selective channel protein 1 (VDAC1) in benign (c) and malignant insulinoma (d); tumor protein D52 (TPD52) in benign (e) and malignant insulinoma (f).

prognostic significance, while others such as CD 99, COX2, and p27 did not. ${ }^{3,13}$

Genetic abnormalities with a clear impact on tumor progression and metastatic potential are rare. In general, non-functioning pancreatic neuroendocrine tumors harbor higher numbers of chromosomal gains and losses than functioning tumors. ${ }^{14}$ The same finding was reported for insulinomas. ${ }^{15}$ Several loci for candidate genes that appeared to be involved in the progression of pancreatic neuroendocrine tumors, such as the loss of $3 p$, have been identified ${ }^{15,16,17}$ but not substantiated by further investigations. Interestingly, among the pancreatic neuroendocrine tumors, insulinomas exhibited a smaller number of genomic alterations than other pancreatic endocrine tumors. Particularly, losses on chromosomes $3 p$ and $6 q$, as well as other malignancy-associated alterations were rare in insulinomas. In a recent genome-wide study in a series of non-familial pancreatic neuroendocrine tumors, MEN1, DAXX, and ATRX were identified as the most commonly mutated genes. However, this study did not assess insulinomas. ${ }^{18}$

As promising as these genetic studies are, none of them have so far been able to provide accurate prognostic marker patterns. In this situation, we conducted a study that took advantage of the proteomic technique, which had not yet been applied to pancreatic neuroendocrine tumors. In order to focus on a clinically and biologically welldefined group of pancreatic neuroendocrine tumors, we restricted our investigations to insulinomas for two reasons: first, most studies on pancreatic neuroendocrine tumors assess the whole phenotypic spectrum, ie, hormonally active tumors, hormonally inactive but hormone-expressing tumors, and also those that completely lack hormones. This approach may lead to important results regarding clinical management and prognostic stratifications of endocrine tumors; however, it probably includes many confounders in the data evaluation and reduces the statistical power when looking for 

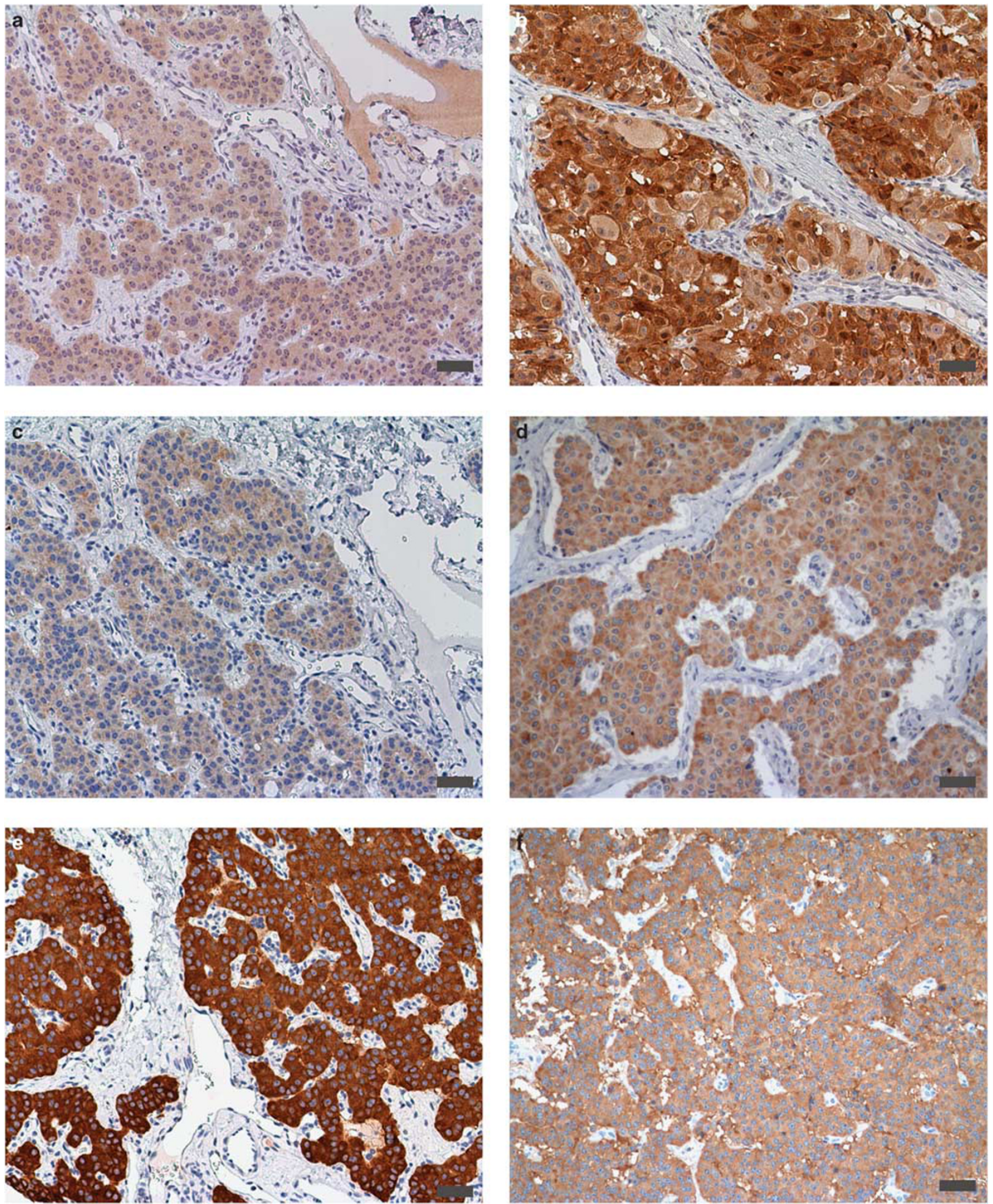

Figure 2 (a) Aldehyde dehydrogenase 1A1 (ALDH1) expression revealed by proteome analysis of six benign and six malignant insulinomas. The data were quantified using DeCyder software; (b) semiquantitative analysis (score 0-12) of ALDH1A1 immunohistochemical stains in 51 benign and 11 malignant insulinomas. $P$-value refers to the Mann-Whitney $U$-test; (c) proteomic analysis of voltage-dependent anion-selective channel protein 1 (VDAC1) and (d) results of VDAC1 immunohistochemistry, (e) Proteomic analysis of tumor protein D52 (TPD52) and (f) results of TPD52 immunohistochemistry; bars mark $100 \mu \mathrm{m}$. 


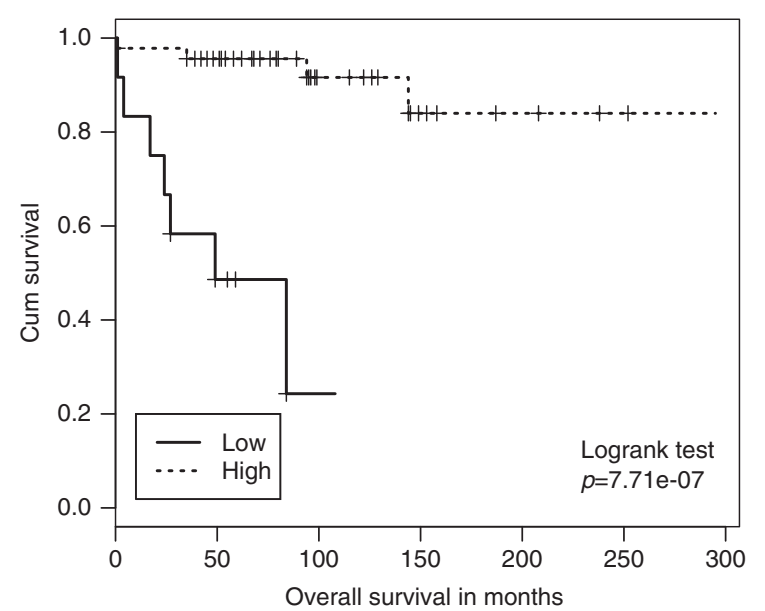

\begin{tabular}{|l|c|c|c|c|c|c|c|}
\hline Low (Score 0-2) & 12 & 5 & 1 & 0 & 0 & 0 & 0 \\
\hline High (Score 3) & 46 & 37 & 17 & 7 & 4 & 2 & 0 \\
\hline
\end{tabular}

Figure 3 Kaplan-Meier curves demonstrating significantly shorter overall survival of patients with low tumor protein D52 (TPD52) levels in their insulinomas. The $P$-value refers to results of log rank analysis.

biologically relevant differentially expressed proteins. Second, the size of the discovery cohort has to be optimized when using proteomics methods due to the limited availability of fresh-frozen tissue, necessitating additional homogenous tumor groups. In the discovery set we searched for proteins that are differentially expressed in benign and malignant insulinomas, and managed to identify 16 differentially expressed proteins. We chose ALDH1A1, VDAC1, TPD52, Gelsolin, and TARDBP for further analysis, because they appeared functionally interesting and could be validated by immunohistochemistry. Logistic regression analysis of the immunohistochemical expression pattern showed that three of the five proteins are good predictors of insulinoma biology.

ALDH1A1 was expressed to a much greater extent in malignant than in benign insulinomas. ALDH1A1 has a role in the formation of retinoic acid, ${ }^{19}$ a potent modulator for gene expression and tissue differentiation, and showed increased activity in both normal and malignant stem cell populations. ${ }^{20}$ ALDH1A1 is therefore thought to be involved in the initiation and maintenance of tumors, and may distinguish cancer stem cells. The mechanism of ALDH1A1 overexpression in cancer cells remains elusive. SOX2 and Nicastrin have been reported to upregulate ALDH1A1 in pancreatic ${ }^{21}$ and breast adenocarcinoma $^{22}$ cells, respectively. Knocking down BRCA1 in breast cancer models is associated with ALDH1A1 upregulation and progenitor cell phenotype. ${ }^{23}$ Recently, the importance of ALDH1A1 for differentiating endocrine precursor cells in pancreas was reported. ${ }^{24,25}$ In our cohort, ALDH1A1 expression was relatively homogeneous within individual insulinomas (instead of a 'stem cell' pattern), indicating an early clonal selection towards ALDH1A1-overexpressing cells in malignant insulinomas.

We also found that VDAC1 is overexpressed in malignant insulinomas compared with benign ones. VDAC1 was identified as a mitochondrial outer membrane stabilizer that functions by forming pores. ${ }^{26}$ VDAC1 is essential for mitochondrial ATP production as a metabolite transporter and serves as a docking site for hexokinase. The VDAC1-bound hexokinase acts as a growth promoter using several mechanisms: (i) VDAC1-hexokinase complex increases the energy and metabolite production in cancer cells; ${ }^{27}$ (ii) VDAC1-hexokinase prevents apoptosis, partly by inhibiting $\mathrm{Bax}$ or $\mathrm{Bak}{ }^{28}$ (iii) mitochondria-associated hexokinase decreases generation of reactive oxygen species, protecting against oxidant-induced cell death; ${ }^{29}$ (iv) mitochondria-associated hexokinase may increase the cholesterol content in mitochondria, which is frequently observed in cancer cells. ${ }^{28}$ Overexpression of VDAC1 has been observed in tumors, including non-small cell lung cancer, ${ }^{30}$ melanoma, and prostate carcinoma cell lines. ${ }^{31}$ The cause of VDAC1 upregulation in human tumors is unclear as yet. The impact of VDAC1 on insulinoma progression is reinforced by the higher expression levels that we found in metastases compared with their primaries. In addition, VDAC1 may serve as a target for pharmacological inhibition, as described for Fluoxetine (Prozac), Cisplatin, Erastin, and Endostatin (for a review, see Shoshan-Barmatz and Mizrachi ${ }^{26}$ ). Some of these compounds have already been introduced in the clinic, providing the opportunity for experimental therapeutic options for incurable insulinoma patients.

TPD52 showed a strikingly higher expression in benign than in malignant insulinomas. TPD52 was linked to the regulation of vesicle trafficking and exocytotic secretion through binding to integral membrane and membrane-associated proteins. TPD52 is related to cell proliferation and apoptosis, and may initiate metastatic phenotype. ${ }^{32,33}$ Overexpression of TPD52 has been described in malignant tumors such as ovarian, prostate and breast cancer, and testicular germ cell tumors. ${ }^{34,35}$ As TPD52 was strongly expressed not only in benign insulinomas but also in normal islets, malignant insulinomas probably undergo loss of TPD52 during neoplastic transformation. At present, it is unclear how this finding relates to the malignant potential of insulinomas.

Multivariate analysis revealed low TPD52 expression to be the strongest independent indicator of malignancy, followed by high ALDH1A1 expression. Remarkably, classical prognostic parameters of pancreatic neuroendocrine tumors did not turn out to be instrumental in this regard, as tumor size showed only borderline significance $(P=0.049)$ and the grade was completely unrelated to malignant behavior. The relevance of downregulation of TPD52 is further emphasized by the fact that low TPD52 expression was very strongly associated with short 
recurrence-free and disease-related survival. Thus, assessing TPD52 expression even has the potential to aid the prognostic stratification of insulinomas in the routine surgical pathology.

In conclusion, the three proteins ALDH1A1, VDAC1, and TPD52 fulfill the requirements for biologic markers that can be used beyond European Neuroendocrine Tumour Society and World Health Organization criteria for prognostic stratification of resected insulinomas. To our knowledge, these are the first protein markers that have been described in a well-defined group of pancreatic neuroendocrine tumors. In the light of these results, it seems promising to further validate the identified proteins and associated processes in pancreatic neuroendocrine tumors, as well as to extend proteome expression studies to other functioning and nonfunctioning pancreatic neuroendocrine tumors in order to find new biological markers for their prognostic assessment.

\section{Acknowledgments}

We thank Sabine Roggenbrodt and Maike Pacena for their excellent technical assistance. We are also grateful to Teresa Gehrs and Kay Dege for proofreading the manuscript. AS is supported by the Associazione Italiana Ricerca Cancro (grant 5 per mille n. 12182) and the Italian Ministry of University and Research (FIRB RBAP10AHJB). AP is supported by the Swiss National Foundation (SNF 310030_144236).

\section{Disclosure/conflict of interest}

The authors declare no conflict of interest.

\section{References}

1 Bosman FT, Carneiro F, Hruban RH, Theise ND (eds). WHO Classification of Tumours of the Digestive System. Chapter 124th edn. International Agency for Research on Cancer: Lyon, 2010.

2 Klöppel G, Couvelard A, Perren A, et al. ENETS Consensus Guidelines for the Standards of Care in Neuroendocrine Tumors: towards a standardized approach to the diagnosis of gastroenteropancreatic neuroendocrine tumors and their prognostic stratification. Neuroendocrinology 2009;90:162-166.

3 Schmitt AM, Anlauf M, Rousson V, et al. WHO 2004 criteria and CK19 are reliable prognostic markers in pancreatic endocrine tumors. Am J Surg Pathol 2007;31:1677-1682.

4 Anlauf M, Gerlach P, Schott M, et al. Pathology of neuroendocrine neoplasms. Chir Z Für Alle Geb Oper Medizen 2011;82:567-573.

5 De Herder WW, Niederle B, Scoazec J-Y, et al. Well-differentiated pancreatic tumor/carcinoma: insulinoma. Neuroendocrinology 2006;84:183-188.

6 Sitek B, Lüttges J, Marcus K, et al. Application of fluorescence difference gel electrophoresis saturation labelling for the analysis of microdissected precursor lesions of pancreatic ductal adenocarcinoma. Proteomics 2005;5:2665-2679.

7 Klose J, Kobalz U. Two-dimensional electrophoresis of proteins: an updated protocol and implications for a functional analysis of the genome. Electrophoresis 1995;16:1034-1059.

8 Nesterenko MV, Tilley M, Upton SJ. A simple modification of Blum's silver stain method allows for 30 minute detection of proteins in polyacrylamide gels. J Biochem Biophys Methods 1994;28:239-242.

9 Sitek B, Sipos B, Pfeiffer K, et al. Establishment of 'onepiece' large-gel 2-DE for high-resolution analysis of small amounts of sample using difference gel electrophoresis saturation labelling. Anal Bioanal Chem 2008;391:361-365.

10 Raffel A, Krausch M, Roushan K, et al. Global histone modification pattern predicts poor prognosis in organic hyperinsulinism. Horm Metab Res 2011;43:858-864.

11 Sitek B, Sipos B, Alkatout I, et al. Analysis of the pancreatic tumor progression by a quantitative proteomic approach and immunhistochemical validation. J Proteome Res 2009;8:1647-1656.

12 Klöppel G. Classification and pathology of gastroenteropancreatic neuroendocrine neoplasms. Endocr Relat Cancer 2011;18(Suppl 1):S1-16.

13 Pelosi G, Fraggetta F, Sonzogni A, Fazio N, Cavallon A, Viale G. CD99 immunoreactivity in gastrointestinal and pulmonary neuroendocrine tumours. Virchows Arch Int J Pathol 2000;437:270-274.

14 Rigaud G, Missiaglia E, Moore PS, et al. High resolution allelotype of nonfunctional pancreatic endocrine tumors: identification of two molecular subgroups with clinical implications. Cancer Res 2001;61:285-292.

15 Jonkers YMH, Claessen SMH, Perren A, et al. Chromosomal instability predicts metastatic disease in patients with insulinomas. Endocr Relat Cancer 2005;12:435-447.

16 Speel EJ, Richter J, Moch H, et al. Genetic differences in endocrine pancreatic tumor subtypes detected by comparative genomic hybridization. Am J Pathol 1999; 155:1787-1794.

17 Barghorn A, Komminoth P, Bachmann D, et al. Deletion at 3p25.3-p23 is frequently encountered in endocrine pancreatic tumours and is associated with metastatic progression. J Pathol 2001;194:451-458.

18 Jiao Y, Shi C, Edil BH, et al. DAXX/ATRX, MEN1, and mTOR pathway genes are frequently altered in pancreatic neuroendocrine tumors. Science 2011;331: 1199-1203.

19 Bhat PV, Poissant L, Wang XL. Purification and partial characterization of bovine kidney aldehyde dehydrogen ase able to oxidize retinal to retinoic acid. Biochem Cell Biol 1996;74:695-700.

20 Ma I, Allan AL. The role of human aldehyde dehydrogenase in normal and cancer stem cells. Stem Cell Rev 2011;7:292-306.

21 Herreros-Villanueva M, Zhang J-S, Koenig A, et al. SOX2 promotes dedifferentiation and imparts stem cell-like features to pancreatic cancer cells. Oncogenesis 2013;2:e61.

22 Lombardo Y, Filipović A, Molyneux G, et al. Nicastrin regulates breast cancer stem cell properties and tumor growth in vitro and in vivo. Proc Natl Acad Sci USA 2012;109:16558-16563.

23 Liu S, Ginestier C, Charafe-Jauffret E, et al. BRCA1 regulates human mammary stem/progenitor cell fate. Proc Natl Acad Sci USA 2008;105:1680-1685. 
24 Matsuda H, Parsons MJ, Leach SD. Aldh1-expressing endocrine progenitor cells regulate secondary islet formation in larval zebrafish pancreas. PLoS One 2013;8:e74350.

25 Li J, Feng ZC, FS-H Yeung, et al. Aldehyde dehydrogenase 1 activity in the developing human pancreas modulates retinoic acid signalling in mediating islet differentiation and survival. Diabetologia 2014;57: 754-764.

26 Shoshan-Barmatz V, Mizrachi D. VDAC1: from structure to cancer therapy. Front Oncol 2012;2:164.

27 Pedersen PL. Warburg, me and Hexokinase 2: Multiple discoveries of key molecular events underlying one of cancers' most common phenotypes, the 'Warburg Effect', i.e., elevated glycolysis in the presence of oxygen. J Bioenerg Biomembr 2007;39:211-222.

28 Pastorino JG, Hoek JB. Regulation of hexokinase binding to VDAC. J Bioenerg Biomembr 2008;40: 171-182.

29 Sun L, Shukair S, Naik TJ, Moazed F, Ardehali H. Glucose phosphorylation and mitochondrial binding are required for the protective effects of hexokinases I and II. Mol Cell Biol 2008;28:1007-1017.
30 Grills C, Jithesh PV, Blayney J, Zhang S-D, Fennell DA. Gene expression meta-analysis identifies VDAC1 as a predictor of poor outcome in early stage non-small cell lung cancer. PLoS One 2011;6:e14635.

31 Lai JC, Tan W, Benimetskaya L, Miller P, Colombini M, Stein CA. A pharmacologic target of G3139 in melanoma cells may be the mitochondrial VDAC. Proc Natl Acad Sci USA 2006;103:7494-7499.

32 Boutros R, Fanayan S, Shehata M, Byrne JA. The tumor protein D52 family: many pieces, many puzzles. Biochem Biophys Res Commun 2004;325:1115-1121.

33 Lewis JD, Payton LA, Whitford JG, et al. Induction of tumorigenesis and metastasis by the murine orthologue of tumor protein D52. Mol Cancer Res 2007;5: 133-144.

34 Byrne JA, Maleki S, Hardy JR, et al. MAL2 and tumor protein D52 (TPD52) are frequently overexpressed in ovarian carcinoma, but differentially associated with histological subtype and patient outcome. BMC Cancer 2010;10:497.

35 Rubin MA, Varambally S, Beroukhim R, et al. Overexpression, amplification, and androgen regulation of TPD52 in prostate cancer. Cancer Res 2004;64:3814-3822.

Supplementary Information accompanies the paper on Modern Pathology website (http://www.nature.com/ modpathol) 